\title{
INTEGRAL THEOREMS IN THREE-DIMENSIONAL POTENTIAL FLOW
}

R. v. MISES

1. Introduction. A potential flow can be described as a vector distribution $\bar{q}(\bar{r})$ ( $\bar{q}=$ velocity vector, $\bar{r}=$ position vector) subject to the two conditions

$$
\operatorname{div} \bar{q}=0, \quad \operatorname{curl} \bar{q}=0 .
$$

Instead of (1) one can also ask that a scalar function $\phi(\bar{r})$ exist so that

$$
\bar{q}=\operatorname{grad} \phi, \quad \Delta \phi=0 .
$$

If both $\bar{q}$ and $\bar{r}$ are restricted to two dimensions, a third form of representation is possible. One can combine the components $x, y$ of $\bar{r}$ and $u, v$ of $\bar{q}$ to two complex numbers

$$
x+i y=\zeta, \quad u-i v=v
$$

and then state that $v$ is an analytic function of $\zeta$. In this case, the Cauchy formula holds,

$$
\int f(v, \zeta) d \zeta=0,
$$

if $f$ is an analytic function of $v$ and $\zeta$ and the integral is extended over the complete boundary of a region in which $f$ is regular.

In the dynamics of the two-dimensional potential flow several equations of the form (4) play a decisive role. It must be expected that the analogous theorems are valid in three-dimensional potential flow also. But the question has not yet been answered: For what vector functions $\bar{f}$ of $\bar{q}$ and $\bar{r}$ is the equation

$$
\int \bar{f}(\bar{q}, \bar{r}) \cdot d \bar{S}=0
$$

correct if the integral is extended over the complete boundary of a region in which $\bar{f}$ has continuous derivatives of the first order with respect to the six components $x, y, z$ of $\bar{r}$ and $u, v, w$ of $\bar{q}$ ? Here, obviously, $d \bar{S}$ is the vectorial area element whose direction is that of the outward normal, and the dot means scalar multiplication. The surface may consist of a finite number of analytic pieces.

The main results of this paper were presented in an address delivered at the New York meeting of the Society on April 4, 1942, by invitation of the Program Committee; received by the editors May 19, 1944. 
The present note will answer this and some related questions and illustrate the applications which can be made in the theory of threedimensional potential flow.

2. The general form of the function $\bar{f}(\bar{q}, \bar{r})$. As $\bar{q}$ is a function of $\bar{r}$ according to (1), one may write $\bar{f}(\bar{q}, \bar{r})=\bar{F}(\bar{r})$. Then the necessary and sufficient condition for (5) being correct for all surfaces is

$$
\operatorname{div} \bar{F}=0 .
$$

If $f_{x}, f_{y}, f_{z}$ denote the components of $\bar{f}$ we find by differentiation:

$$
\begin{gathered}
\operatorname{div} F=\frac{\partial f_{x}}{\partial x}+\frac{\partial f_{y}}{\partial y}+\frac{\partial f_{z}}{\partial z}+\frac{\partial u}{\partial x} \frac{\partial f_{x}}{\partial u}+\frac{\partial v}{\partial x} \frac{\partial f_{x}}{\partial v}+\frac{\partial w}{\partial x} \frac{\partial f_{x}}{\partial w} \\
+\frac{\partial u}{\partial y} \frac{\partial f_{y}}{\partial u}+\frac{\partial v}{\partial y} \frac{\partial f_{y}}{\partial v}+\cdots+\cdots
\end{gathered}
$$

It can be seen easily that the right-hand expression is zero, by virtue of (1), if the following conditions are fulfilled:

$$
\begin{gathered}
\partial f_{x} / \partial x+\partial f_{y} / \partial y+\partial f_{z} / \partial z=0 \\
\partial f_{x} / \partial u=\partial f_{y} / \partial v=\partial f_{z} / \partial w \\
\partial f_{z} / \partial v+\partial f_{y} / \partial w=\partial f_{x} / \partial w+\partial f_{z} / \partial u=\partial f_{y} / \partial u+\partial f_{x} / \partial v=0 .
\end{gathered}
$$

On the other hand, these conditions are also necessary, as the following examples show. First, take $u=1, v=1, w=1$, then condition (a) follows. Second, take $u=x, v=-y, w=0$ in accordance with (1), then the first equality (b) follows. Finally, choose $u=y, v=x$, $w=0$ which also fulfills (1), then the last equality (c) appears as necessary. Thus, the three components $f_{x}, f_{y}, f_{z}$ have to satisfy the six differential equations (a), (b), (c).

The five equations (b) and (c) express the fact that the infinitesimal transformation of the $\bar{q}$-space, given by

$$
d \bar{q} / d t=\bar{f}(\bar{q}, \bar{r})
$$

for any constant $\bar{r}$ ( $t=$ scalar parameter), is conformal (angle preserving). Without using this fact the general solution of the equations (b) and (c) can be found in the following way.

Assume that $f_{x}, f_{y}, f_{z}$ are developable into a power series with respect to $u, v, w$ within some finite region. Then (b) and (c) furnish relations between the coefficients of terms of the same order $n$. There are three times $(n+1)(n+2) / 2$ such coefficients and five times $n(n+1) / 2$ relations. It can be seen that these relations are linearly 
independent of each other. The excess of the number of coefficients over the number of equations is

$$
\begin{gathered}
e=3(n+1)(n+2) / 2-5 n(n+1) / 2=(n+1)(3-n), \\
\text { that is, } e=3,4,3,0,<0 \\
\text { for } n=0,1,2,3,>3 .
\end{gathered}
$$

Consequently, there exist $\infty^{3}$ solutions of order zero, $\infty^{4}$ solutions of order 1, and $\infty^{3}$ solutions of order 2 and no other ones. All these solutions can be found by setting up the linear equations for the coefficients, and so on. But, at least for $n=0$ and $n=1$, they are obvious:

For $n=0$ we have the translations

$$
\bar{f}=\bar{A}_{0}
$$

where the vector $\bar{A}_{0}$ is independent of $\bar{q}$. For $n=1$ one has the homography and the rotation

$$
f=K \bar{q}+(\bar{A} \times \bar{q})
$$

where the scalar $K$ and the vector $\bar{A}$ are functions of $\bar{r}$ alone (cross denoting the vector multiplication). For $n=2$ one may find by computation or by certain geometrical considerations

$$
f=\bar{L} q^{2}-2(\bar{L} \cdot \bar{q}) \bar{q}
$$

where $q^{2}=u^{2}+v^{2}+w^{2}$ and $\bar{L}$ is a vector depending on $\bar{r}$ but not on $\bar{q}$. Each infinitesimal transformation of this group consists of an inversion, an infinitesimal translation in direction of $\bar{L}$, and another inversion.

The general, ten-parametric, expression for $\bar{f}$ is thus

$$
\bar{f}=\bar{A}_{0}+K \bar{q}+(\bar{A} \times \bar{q})+\bar{L} q^{2}-2(\bar{L} \cdot \bar{q}) \bar{q} .
$$

This expression has now to satisfy the condition (a) independently of $\bar{q}$. For $\bar{A}_{0}, K, \bar{A}$ one finds immediately

$$
\begin{aligned}
\operatorname{div} \bar{A}_{0} & =0, \\
\operatorname{grad} K+\operatorname{curl} \bar{A} & =0, \cdots \quad(\Delta K=0),
\end{aligned}
$$

while $\bar{L}$ has to fulfill the six equations

$$
\begin{gathered}
\partial L_{x} / \partial x=\partial L_{y} / \partial y=\partial L_{z} / \partial z=0 \\
\partial L_{z} / \partial y+\partial L_{y} / \partial z=\partial L_{x} / \partial z+\partial L_{z} / \partial x=\partial L_{y} / \partial x+\partial L_{x} / \partial y=0
\end{gathered}
$$

This means that the space transformation $d \bar{r} / d t=\bar{L}$ is angle preserving and length preserving. Thus 


$$
\bar{L}=\bar{L}_{0}+\left(\bar{L}_{1} \times \bar{r}\right)
$$

with $\bar{L}_{0}$ and $\bar{L}_{1}$ constants.

The result can be stated: The most general function $\bar{f}(\bar{q}, \bar{r})$ which satisfies (5) for an arbitrary surface $S$ and any $\bar{q}(\bar{r})$ fulfilling (1) is given by (10) provided the functions $\bar{A}_{0}, K, \bar{A}$, and $\bar{L}$ of $\bar{r}$ satisfy (11), (12), and (13) with $\bar{L}_{0}$ and $\bar{L}_{1}$ constants.

3. Simple examples. The first term, $\bar{A}_{0}$, in the expression for $\bar{f}$ which is independent of $\bar{q}$ can be disregarded.

The simplest linear function is determined by $K=$ const., $\bar{A}=0$. This gives nothing else than the integral form of the divergence condition:

$$
\int \bar{q} \cdot d \bar{S}=\int(\bar{q} \cdot \bar{n}) d S=\int q_{n} d S=0 .
$$

Here and in the following, $\bar{n}$ is the unit vector in the direction of the outward normal and the subscript $n$ refers to the vector component in this direction.

If $K=0$ and $\bar{A}=$ const. one finds, using the formula $(\bar{A} \times \bar{q}) \cdot \bar{n}$ $=(\bar{q} \times \bar{n}) \cdot \bar{A}$, that each component of

$$
\int(\bar{q} \times \bar{n}) d S=0
$$

is justified. This vector integral may be considered as a surface circulation. If it is extended over a surface surrounding a rigid body (that is, not over the complete boundary of a region of regular $\bar{q}$ ), it may have a constant value different from zero. If the region outside the body includes a discontinuity surface $S^{\prime}$, one has to add the integral of $\left(\bar{q}_{1}-\bar{q}_{2}\right) \times \bar{n}^{\prime}$, extended over $S^{\prime}$ where $\bar{n}^{\prime}$ is the unit vector of the normal to $S^{\prime}$ in the direction from 1 to 2 , and $\bar{q}_{1}$ and $\bar{q}_{2}$ are the velocities on both sides. This vector $\left(\bar{q}_{1}-\bar{q}_{2}\right) \times \bar{n}^{\prime}$ has the direction of the vortex line and the magnitude of the vortex density on $S^{\prime}$ if the discontinuity surface is regarded as a vortex sheet. In a field of vortex motion the left-hand side of (15) equals the volume integral of $-\operatorname{curl} \bar{q}$.

As a next step one may take, with $K=0$, for $\bar{A}$ some gradient, for example a function $g(r) \bar{r}$. Then the formulae

$$
\int g(r)(\bar{r} \times \bar{q}) \cdot d \bar{S}=\int g(r)(\bar{q} \times \bar{n}) \cdot \bar{r} d S=0
$$

result. These integrals are zero even when extended over a surface 
surrounding an obstacle since they vanish for the spheres $r=$ const. It is understood that singular points of $g(r)$ have to be excluded.

If $K$ is chosen to equal $x$, the vector $\bar{A}$ must be (except for a gradient which always can be added) $\bar{r} \times \bar{i}_{x} / 2$ where $i_{x}$ is the unit vector in $x$-direction. Combining this with the analogous formulae for $y$ and $z$, the following vector equation results:

$$
\begin{aligned}
\int[\bar{r}(\tilde{q} \cdot \bar{n})-(\bar{r} / 2) & \times(\bar{q} \times \tilde{n})] d S \\
& =\int[\bar{r}(\bar{q} \cdot \bar{n})-\bar{q}(\bar{r} \cdot \bar{n}) / 2+\bar{n}(\bar{r} \cdot \bar{q}) / 2 S d]=0 .
\end{aligned}
$$

This may be considered as a relation for the moment of surface circulation.

If any spherical harmonic is taken for $K$, it is easy to find a corresponding vector $\bar{A}$. Two examples may suffice:

$$
K=x y, \quad A_{x}=0, \quad A_{y}=0, \quad A_{z}=\left(y^{2}-x^{2}\right) / 2
$$

and

Again, one may combine three expressions of each kind and thus form a vector integral, like (17), which vanishes when taken over the complete boundary of a region of regularity.

4. Cauchy formula. In the two-dimensional case the value of any analytic function of $x+i y$ and $u-i v$ in an arbitrary point can be expressed in terms of its values on a curve surrounding this point. This is based on a formula of the type (4) where the $f$ has such a singularity at $\zeta=0$ that the integral over a circle of radius $\rho$ has a finite limit when $\rho$ tends toward zero. To achieve this kind of singularity one has simply to take $f(v, \zeta)=g(v, \zeta) / \zeta$ where $g$ is regular in $\zeta=0$, since

$$
\lim _{\rho=0} \int_{c_{\rho}} \frac{d \zeta}{\zeta}=2 \pi i
$$

(where $C_{\rho}$ is the circle $|\zeta|=\rho$ ). Then, if $g$ is continuous at $\zeta=0$,

$$
\lim _{\rho=0} \int \frac{g(v, \zeta) d \zeta}{\zeta}=2 \pi i g(0, v[0]) \text {. }
$$

In order to obtain analogous equations in the three-dimensional case we have to look for functions $\bar{f}$ such that $f r^{2}$ remains finite when $r$ 
tends to zero. It is seen from the general result in $\$ 2$ that this can never be the case with a term of second order in $\bar{q}$ since its coefficient is linear in $\bar{r}$. We omit the case of order zero terms, that is, of terms which do not involve $\bar{q}$. Thus only the terms $K \bar{q}$ and $\bar{A} \times \bar{q}$ are left where $K$ must be a harmonic function according to (12).

The only harmonic functions which behave like $1 / r^{2}$ at the origin are $x / r^{3}, y / r^{3}, z / r^{3}$. Taking the first for $K$ one can easily find from equation (12) the components of $A$ (as in the examples (18), (19)):

$$
K=x / r^{3}, \quad A_{x}=0, \quad A_{y}=-z / r^{3}, \quad A_{z}=y / r^{3} .
$$

The components of $K \bar{q}+(\bar{A} \times \bar{q})$ are then, except for the factor $1 / r^{\mathbf{3}}$ :

$$
x u-y v-z w, \quad x v+y u, \quad x w+z u
$$

and, if $y / r^{3}$ and $z / r^{3}$ are taken for $K$ :

$$
\begin{array}{llr}
y u+x v, & y v-z w-x u, & y w+z v, \\
z u+x w, & z v+y w, \quad z w-x u-y v .
\end{array}
$$

If each of the three vectors represented by $(23)$ and $\left(23^{\prime}\right)$ is multiplied by $\bar{n}$, the three scalar products form the components of one vector which can be considered as a particular triple product of $\bar{r}, \bar{q}$, and $\bar{n}$. This product may be designated by $(\bar{r}, \bar{q} ; \bar{n})$ and can be expressed in three different forms by combinations of the usual products:

$$
\begin{aligned}
(\bar{r}, \bar{q} ; \bar{n}) & =\bar{r}(\bar{q} \cdot \bar{n})+\bar{q}(\bar{r} \cdot \bar{n})-\bar{n}(\bar{q} \cdot \bar{r}) \\
& =\bar{r}(\bar{q} \cdot \bar{n})+\bar{r} \times(\bar{q} \times \bar{n}) \\
& =\bar{q}(\bar{r} \cdot \bar{n})+\bar{q} \times(\bar{r} \times \bar{n}) .
\end{aligned}
$$

On a sphere $r=$ const., where $\bar{n}=\bar{r} / r$, this reduces to $r \bar{q}$, as the last expression shows. Therefore, the integral over an infinitesimal sphere reduces to

$$
\lim _{\rho=0} \int_{\left(S_{\rho}\right)} \frac{r \bar{q}}{r^{3}} d S=\bar{q}(0) \int_{\left(S_{\rho}\right)} \frac{d S}{r^{2}}=4 \pi \bar{q}(0)
$$

and the Cauchy formula for the three-dimensional space reads

$$
\bar{q}(0)=\frac{1}{4 \pi} \int \frac{(\bar{r}, \bar{q} ; \bar{n})}{r^{3}} d S,
$$

where the integral has to be extended over a surface surrounding the origin and including no singular point of the velocity distribution. ${ }^{1}$

1 This formula has already been given by Fulton and Rainich (Amer. J. Math. vol. 54 (1932) pp. 235-241). See also S. Bergman, Bull. Amer. Math. Soc. vol. 49 (1943) p. 174. 
The equation (26) is the only formula of Cauchy type involving the velocity of a potential flow in three dimensions. It follows from the preceding that for no other function of $\bar{r}$ and $\bar{q}$, for example the products $\bar{r} \cdot \bar{q}$ and $\bar{r} \times \bar{q}$, its value in an arbitrary point can be computed from the values the function assumes on a surrounding surface. This, however, is only seemingly less general than what is the case in the two-dimensional problem. Here, any analytic function of $v$ and $\zeta$ can be computed in the form (21). But giving the values of $g(v, \zeta)$ at all points of the contour is the same as giving here $v$, while in space the knowledge of a function, for example $\bar{r} \times \bar{q}$, is not sufficient to determine $\bar{q}$. If $\bar{q}$ is given in all points of $S$, equation (26) supplies the values of any function of $\bar{r}$ and $\bar{q}$ in an arbitrary point (within the range of regularity).

The matrix formed by the nine quantities (22) and $\left(22^{\prime}\right)$ can be considered as a symmetric tensor by which the unit vector $\bar{n}$ has to be multiplied. Using Gibbs' notation of dyadics and the sign $I$ for the unit tensor, one can write this tensor as

$$
(\bar{r} ; \bar{q})+(\bar{q} ; \bar{r})-I(\bar{r} \cdot \bar{q})
$$

or, applying Jaumann's dyadics also, as

$$
(\bar{r} ; \bar{q})+(\bar{r} \times \bar{q}) \text {. }
$$

The linear transformation determined by this tensor consists of the reflection with respect to the bisectrix of $\bar{r}$ and $\bar{q}$ and a contraction at the rate $\cos (\bar{r}, \bar{q})$ in the direction normal to the plane extended by $\bar{r}$ and $\bar{q}$. In the two-dimensional case the reflection only remains, which is easily expressed in terms of complex numbers.

5. Biot-Savart formula. The Cauchy formula (26) shall now be applied to the following case. We consider the space included in a sphere of infinite radius. The velocity at infinity has the constant value $\bar{q}_{\infty}$. We admit a finite number of discontinuity surfaces $S_{1}, S_{2}, S_{3}, \ldots$ which, of course, are tangential to the velocity vector, that is, $\bar{q} \cdot \bar{n}=0$ on both sides of each $S_{\nu}$. Some of the $S_{\nu}$ may be closed surfaces, in the inner of which $\bar{q}$ must necessarily vanish. Such $S_{\nu}$ represent rigid bodies (obstacles) that are surrounded by the flow.

If any point not on a discontinuity surface is chosen as origin, equation (26) will give its velocity vector $\bar{q}(0)$, if the integral is extended over the infinite sphere and both sides of each discontinuity surface. The integral over the sphere supplies $4 \pi \bar{q}_{\infty}$, according to what was said in connection with (25). On a closed $S_{\nu}$ the integral for the inner side is zero, since here $\bar{q}=0$. Denoting now by $\bar{n}$ the outward 
normal of the body (=inside normal of the fluid mass), we have to reverse the sign of the integrand in (26) which then reads, according to $\bar{q} \cdot \bar{n}=0$ and the second expression (24), $(\bar{q} \times \bar{n}) \times \bar{r} / r^{3}$. On each open discontinuity surface, $\bar{q}$ may refer to the side toward which $\bar{n}$ points. Then (26) becomes

$$
\bar{q}(0)=\bar{q}_{\infty}+\frac{1}{4 \pi} \int \frac{(\bar{q} \times \bar{n}) \times \bar{r}}{r^{3}} d S,
$$

where the integral has to be extended over both sides of all discontinuity surfaces. If only the outer side of the closed $S_{\nu}$ and one side of the open $S_{\nu}$ is taken and $\bar{q}^{\prime}$ called the velocity on the opposite sides, one can write instead of (28):

$$
\bar{q}(0)=\bar{q}_{\infty}+\frac{1}{4 \pi} \int \frac{\left[\left(\bar{q}-\bar{q}^{\prime}\right) \times \bar{n}\right] \times \bar{r}}{r^{3}} d S,
$$

where $\bar{q}^{\prime}=0$ for the closed $S_{\nu}$ which represent obstacles.

The equations (28) and $\left(28^{\prime}\right)$ are in close analogy to the so-called Biot-Savart formula which-in hydrodynamic terms-gives the velocity "induced" at the point $r=0$ by a "vortex line" of vorticity $\bar{C}$ :

$$
\bar{q}(0)=\frac{1}{4 \pi} \int \frac{\bar{C} \times \bar{r}}{r^{3}} d s,
$$

the integral being taken over the arc length of the (closed) vortex line. Thus, the result $\left(28^{\prime}\right)$ can be stated as follows: The velocity excess $\bar{q}-\bar{q}_{\infty}$ can be considered as induced by vortex sheets extended over all discontinuity surfaces (including the obstacle surfaces), with a vortex-line density of magnitude $\bar{q}-\bar{q}^{\prime}$, directed perpendicular to $\bar{q}-\bar{q}^{\prime}$ in the tangential plane $\left(\bar{q}^{\prime}=0\right.$ on the closed surfaces).

This statement is the basis of the three-dimensional wing theory of Lanchester and Prandtl. Its equivalent was deduced by Prandtl and his followers from certain assumptions about impulsive forces and fictitious mass forces inside the bodies.

The formula (28) or $\left(28^{\prime}\right)$ is valid only if the origin $O$ is not a point of one of the discontinuity surfaces. If $O$ approaches $S$, the limit of the expression on the right-hand side includes, in addition to the integral over $S$, the quantity $\left[\tilde{q}(0)-\bar{q}^{\prime}(0)\right] / 2$, as can be shown easily in the usual way. Therefore $\left(28^{\prime}\right)$ becomes, for $O$ on $S$ :

$$
\left[\bar{q}(0)+\bar{q}^{\prime}(0)\right] / 2=\bar{q}_{\infty}+\frac{1}{4 \pi} \int \frac{\left[\left(\bar{q}-\bar{q}^{\prime}\right) \times \bar{n}\right] \times \bar{r}}{r^{3}} d S,
$$

where again $\bar{q}^{\prime}(0)$ vanishes if the point under consideration falls on a 
closed discontinuity surface. This relation, in connection with the fact that on both sides of a free discontinuity surface the pressure (the velocity amount) must have the same value, leads to the integral equations which determine the vortex distribution on $S$ as well as the shape of those parts of $S$ which constitute the free discontinuity surfaces.

6. The second order terms. Momentum theorems. The last two terms in (10) with $\bar{L}$ satisfying the condition (13) lead to two new integral formulae. First, taking $L_{1}=0$ we have

$$
\int\left[L_{0} q^{2}-2\left(\bar{L}_{0} \cdot \bar{q}\right) \bar{q}\right] \cdot d \bar{S}=\int\left[q^{2}\left(\bar{L}_{0} \cdot \bar{n}\right)-2\left(\bar{L}_{0} \cdot \bar{q}\right)(\bar{q} \cdot \bar{n})\right] d S .
$$

If $\bar{L}_{0}$ is identified with the unit vector in $x$-direction, (31) is the $x$-component of the equation

$$
\int\left[q^{2} \bar{n}-2(\bar{q} \cdot \bar{n}) \bar{q}\right] d S=0 .
$$

The vector $\bar{n}^{\prime}$ defined by

$$
\bar{n}^{\prime}=\bar{n}-2((\bar{q} / q) \cdot \bar{n}) \bar{q} / q
$$

is easily seen to be a unit vector, symmetrical to $\bar{n}$ with respect to the plane normal to $\bar{q}$. Equation (32) can then be written as

$$
\int q^{2} \bar{n}^{\prime} d S=0 .
$$

If, on the other hand, in equation (13), $\bar{L}_{0}$ is taken as zero the integral formula will read

$$
\int\left[\left(\bar{L}_{1} \times \bar{r}\right) q^{2}-\left\{2\left(\bar{L}_{1} \times \bar{r}\right) \cdot \bar{q}\right\} \bar{q}\right] \cdot d \bar{S}=0 .
$$

Setting here $d \bar{S}=\bar{n} d S$ and using the identity $(\bar{a} \times \bar{b}) \cdot \bar{c}=\bar{a} \cdot(\bar{b} \times \bar{c})$, equation (34) becomes

$$
\int\left\{q^{2} \bar{L}_{1} \cdot(\bar{r} \times \bar{n})-2\left[\bar{L}_{1} \cdot(\bar{r} \times \bar{q})\right](\bar{q} \cdot \bar{n})\right\} d S=\int q^{2} \bar{L}_{1} \cdot\left(\bar{r} \times \bar{n}^{\prime}\right) d S=0 .
$$

This is, if $\bar{L}_{1}$ is considered as the unit vector in $x$-direction, the $x$-component of the equation

$$
\int \bar{r} \times\left[q^{2} \bar{n}-2(\bar{q} \cdot \bar{n}) \bar{q}\right] d S=0
$$


or using (33)

$$
\int q^{2}\left(\bar{r} \times \bar{n}^{\prime}\right) d S=0
$$

The two relations $\left(32^{\prime}\right)$ and $\left(35^{\prime}\right)$ are the three-dimensional generalizations of $\int v^{2} d \zeta=0$ and $\int \zeta v^{2} d \zeta=0$ which are sometimes known as the Blasius formulae. In fact, when applied to a two-dimensional velocity distribution and a cylindrical $S$, our equations $\left(32^{\prime}\right)$ and $\left(35^{\prime}\right)$ coincide with those complex integrals. The argument of the complex number $v^{2} d \zeta$ corresponds to the direction of the "reflected normal" $\bar{n}^{\prime}$.

The physical meaning of the integrals (32) and (35) can easily be understood. If $S$ is the closed surface of a rigid body, $\bar{q} \cdot \bar{n}$ equals zero on $S$ and $(-\rho / 2) q^{2}$ where $\rho$ is the density can be considered as the pressure value. As $\bar{n}$ is the direction of the thrust upon the body, the integrals of $(-\rho / 2) \int q^{2} \bar{n}^{\prime} d S$ and $(-\rho / 2) \int q^{2}\left(\bar{r} \times \bar{n}^{\prime}\right) d S$ give the resultant force and the moment of the fluid reaction upon the body. Thus the equations (32') and (35') allow the following statement: If $S$ is a closed surface the inner of which consists of some rigid bodies with surfaces $S_{1}, S_{2}, S_{3}, \cdots$ and of a fluid mass with finite velocity distribution satisfying (1), then the resultant force $\bar{F}$ and the moment $\bar{M}$ of the fluid reaction upon $S_{1}, S_{2}, S_{3}, \cdots$ are given by

$$
\bar{F}=\frac{\rho}{2} \int q^{2} \bar{n}^{\prime} d S, \quad \bar{M}=\frac{\rho}{2} \int q^{2}\left(\bar{r} \times \bar{n}^{\prime}\right) d S
$$

where $\bar{n}^{\prime}$, at each point of $S$, is the normal vector reflected with respect to the plane perpendicular to $\bar{q}$.

These are evidently the momentum theorems for the reactions upon bodies surrounded by a potential flow. Note that free discontinuity surfaces inside $S$ do not invalidate (36) since $q^{2}$ must have the same value on both sides and $\bar{q} \cdot \bar{n}$ must vanish at each point of such a surface. Another useful form of the momentum theorems would be this: If the complete boundary $S$ of a region of potential flow with finite velocities consists of two parts $S^{\prime}$ and $S^{\prime \prime}$ where $S^{\prime \prime}$ is tangential to the flow, the integrals (36), extended over $S^{\prime}$ only, give the fluid reactions exerted along $S^{\prime \prime}$. This would apply, for example, to the flow through a channel where $S^{\prime \prime}$ consists of the walls of the channel and $S^{\prime}$ of two cross sections, one at the entrance and the other at the exit.

7. D'Alembert's paradox and the generalization of Joukowski's formula. If a rigid body or a group of bodies is moving at constant 
velocity $-\bar{c}$ through a bulk of fluid at rest, the inverse motion, with the bodies at rest and the fluid velocity $\bar{q}_{\infty}=\bar{c}$ at infinity, is a potential flow. Setting

$$
\bar{q}=\bar{q}_{\infty}+\bar{q}^{\prime}=\bar{c}+\bar{q}^{\prime},
$$

$\bar{q}^{\prime}$ as well as $\bar{q}$ fulfill the equations (1) and $\tilde{q}^{\prime}$ vanishes at infinity.

To find the fluid reactions upon the moving bodies we introduce (37) in the expression $q^{2} \bar{n}-2(\bar{q} \cdot \bar{n}) \bar{q}$ of (32):

$$
\begin{aligned}
q^{2} \bar{n}-2(\bar{q} \cdot \bar{n}) \bar{q}= & c^{2} \bar{n}-2(\bar{c} \cdot \bar{n}) \bar{c} \\
& +2\left(\bar{c} \cdot \bar{q}^{\prime}\right) \bar{n}-2(\bar{c} \cdot \bar{n}) q^{\prime}-2\left(\bar{q}^{\prime} \cdot \bar{n}\right) \bar{c} \\
& +q^{\prime 2} \bar{n}-2\left(\bar{q}^{\prime} \cdot \bar{n}\right) \bar{n} .
\end{aligned}
$$

The first terms, depending on $\bar{c}$ only, give no contribution to either of the integrals (32) or (35). The bilinear terms in the second line can be written, according to (24), as

$$
-2\left(\bar{c}, \bar{q}^{\prime} ; \bar{n}\right)=-2 \bar{c}\left(\bar{q}^{\prime} \cdot \bar{n}\right)-2 \bar{c} \times\left(\bar{q}^{\prime} \times \bar{n}\right) .
$$

Let us now assume that $\bar{q}$ can be developed into a power series at $r=\infty$. Then, since $\bar{q}$ is the gradient of a harmonic function corresponding to (2), the first terms are

$$
\begin{aligned}
& \bar{q}=\bar{c}+\operatorname{grad}\left(\frac{a_{0}}{r}+\frac{\bar{a} \cdot \bar{r}}{r^{3}}+\cdots\right), \\
& \bar{q}^{\prime}=\frac{-a_{0} \bar{r}}{r^{3}}+\left[\frac{\bar{a}}{r^{3}}-\frac{3(\bar{a} \cdot \bar{r}) \bar{r}}{r^{5}}\right]+\cdots .
\end{aligned}
$$

The first term in $\bar{q}^{\prime}$ implies a source or sink at infinity. If this is excluded, the lowest terms are of order $r^{-3}$ and that means that the contribution to the integral (32), if it is extended over the sphere $r=\infty$, is zero. Thus the conclusion is reached: If some bodies are moving at constant velocity $-\bar{c}$ through a bulk of fluid at rest and the velocity distribution is supposed to be developable at infinity and no source or sink admitted, then the total air reaction upon the bodies can consist of a couple only, the resultant force being zero.

This statement includes the so-called d'Alembert paradox which states that a sphere moving through a fluid at rest experiences no resultant force under normal conditions. It is seen from our argument that this is still valid if some discontinuity surfaces, not extending to infinity, are present.

If the boundary of one of the rigid bodies or a discontinuity surface extends to infinity, the assumption of a developable $\bar{q}$-distribution is 
not permitted. Then, a resultant force determined by the second and third groups of terms in (38) may exist. It follows from (39), since the integral of $\bar{q}^{\prime} \cdot \bar{n}$ over a closed surface must vanish, that the terms linear in $\bar{q}^{\prime}$ are determined by the product of $2 \bar{c}$ and the integral of $\bar{q}^{\prime} \times \bar{n}$ which is obviously the same as that of $\bar{q} \times \bar{n}$, that is, the surface circulation according to (15). Thus the following conclusion is reached: The resultant force of the fluid reaction upon bodies moving at a constant speed $-\bar{c}$ through a fluid at rest consists of two parts; the first one, linear in $\bar{q}^{\prime}$, is

$$
\bar{F}_{1}=-\rho \bar{c} \times \bar{C}
$$

where $\bar{C}$ is the surface circulation, defined as the left-hand expression in (15); the second, quadratic, part is

$$
F_{2}=\frac{\rho}{2} \int q^{\prime 2} \bar{n}^{\prime} d S
$$

where $\bar{n}^{\prime}$ is the normal vector reflected on the plane perpendicular to $\bar{q}^{\prime}$.

In the two-dimensional case, if there are no discontinuity surfaces, only $\bar{F}_{1}$ exists and the analogy to (41) is known as the Kutta-Joukowski formula. In the case of flow with separation (Helmholtz flow) $\bar{F}_{2}$ is prevailing.- Note that in the three-dimensional problem, $\bar{F}_{1}$ is perpendicular to the velocity vector $\bar{c}$ while, in general, nothing can be said about the direction of $\bar{F}_{2}$.

In the "regular" case, that is, with $\bar{q}$ developable at infinity, the order of magnitude of $\bar{q}^{\prime}$ is $r^{-3}$ and thus the surface circulation $\bar{C}$ as well as the integral in (42) vanish.

Let us, finally, compute the first order terms $\bar{M}_{1}$ (the terms linear in $\bar{q}^{\prime}$ ) in the expression for the moment. From the second equation (36) and the second line in (38) we find

$$
\begin{aligned}
\bar{M} & =-\rho \int \bar{r} \times\left[\left(\bar{q}^{\prime} \cdot \bar{n}\right) \bar{c}+(\bar{c} \cdot \bar{n}) \bar{q}^{\prime}-\left(\bar{c} \cdot \bar{q}^{\prime}\right) \bar{n}\right] d S \\
& =-\rho \int \bar{r} \times\left(\bar{c}, \bar{q}^{\prime} ; \bar{n}\right) d S .
\end{aligned}
$$

If $\bar{q}^{\prime}$ is introduced from (40) with $a_{0}=0$ and a sphere of radius $r$ with $\tilde{n}=\tilde{r} / r$ taken for $S$, one has

$$
\bar{q}^{\prime} \cdot \bar{n}=-2 \bar{a} \cdot \bar{r} / r^{4}, \quad \bar{r} \times \bar{q}^{\prime}=\bar{r} \times \bar{a} / r^{3}, \quad \bar{r} \times \bar{n}=0 .
$$

On the other hand, $\bar{M}_{1}$ can be identified with the total moment $\bar{M}$ since for an infinite $r$ the quadratic term $\bar{M}_{2}$ vanishes. Thus 


$$
\bar{M}=\rho \bar{c} \times \int-2 \frac{\bar{a} \cdot \bar{r}}{r^{4}} \bar{r} d S+\rho \bar{a} \times \int \frac{\bar{c} \cdot \bar{r}}{r^{4}} \bar{r} d S .
$$

It can be seen easily that for any sphere of radius $r$

$$
\int(\bar{a} \cdot \bar{r}) \bar{r} d S=\frac{4 \pi}{3} r^{4} \bar{d}
$$

Therefore

$$
\bar{M}=(4 \pi / 3)[-(2 \bar{c} \times \bar{a})+(\bar{a} \times \bar{c})]=4 \pi(\bar{a} \times \bar{c}) .
$$

Since $\bar{a}$ is proportional to $\bar{q}^{\prime}$ and, other things being equal, $\bar{q}^{\prime}$ must be proportional to $\bar{c}$ we have obtained the result-known in the classical theory: In the regular case, that is, with $\bar{q}^{\prime}$ developable at infinity, the fluid reaction upon bodies moving at constant speed $-\bar{c}$ has a moment vector perpendicular to $\bar{c}$ and proportional to $\rho$ and $c^{2}$.

A more detailed discussion of this and related questions is beyond the scope of the present note.

HARVARD UNIVERSITY 